\title{
A Computational Approach to Explicit Feedback Stochastic Nonlinear Model Predictive Control
}

\author{
Alexandra Grancharova and Tor A. Johansen
}

\begin{abstract}
Nonlinear Model Predictive Control (NMPC) involves the solution at each sampling instant of a finite horizon optimal control problem subject to nonlinear system dynamics, and state and input constraints. Mathematical models of engineering systems usually contain some amount of uncertainty. In the robust NMPC problem formulation, the model uncertainty is taken into account. This paper presents an approximate multi-parametric Nonlinear Programming approach to explicit solution of feedback stochastic MPC problems for constrained nonlinear systems in the presence of stochastic uncertainty. It is assumed that the discrete probability distribution of the uncertainty is known. The mathematical expectation of the cost function is minimized subject to state and input constraints. The approximate explicit approach constructs a piecewise nonlinear approximation to the optimal sequence of feedback control policies. It is demonstrated by explicit feedback stochastic NMPC for a cart moving on a plane and attached to the wall via a spring.
\end{abstract}

\section{INTRODUCTION}

$\mathrm{N}$ ONLINEAR Model Predictive Control (NMPC) involves the solution at each sampling instant of a finite horizon optimal control problem subject to nonlinear system dynamics and state and input constraints [1], [2], [3]. A survey of the numerical methods for on-line solution of NMPC problems is given in [4]. Most recently, an advanced-step NMPC controller with reduced on-line computational delay has been proposed in [5]. Several approaches to explicit solution of NMPC problems have been suggested in the literature. The benefits of an explicit solution, in addition to the efficient on-line computations, include also verifiability of the implementation. In [6], [7], [8], approaches for off-line computation of explicit suboptimal piecewise linear (PWL) predictive controllers for nonlinear systems with state and input constraints have been developed, based on the multi-parametric Nonlinear Programming (mp-NLP) ideas [9]. In [8], computational methods to handle non-convex mp-NLP problems have been suggested. Algorithms for solving mp-NLP problems, including the non-convex case, are described also in [10].

This work was sponsored by the Research Council of Norway through the Strategic University Program on Control, Information and Communication Systems for Environmental and Safety Critical Systems.

A. Grancharova is with the Institute of System Engineering and Robotics, Bulgarian Academy of Sciences, Acad. G. Bonchev str., B1.2, P.O.Box 79, Sofia 1113, Bulgaria (phone: +359889625010; fax: +35928703361; e-mail: alexandra.grancharova@abv.bg).

T. A. Johansen is with the Department of Engineering Cybernetics, Norwegian University of Science and Technology, 7491 Trondheim, Norway (e-mail: Tor.Arne.Johansen@itk.ntnu.no).
Mathematical models of engineering systems usually contain some amount of uncertainty (typically unknown additive disturbances and/or uncertain model parameters). In the robust MPC problem formulation, the model uncertainty is taken into account. In some applications, the system to be controlled is described by a stochastic model where the probabilistic distribution of the uncertainty is assumed to be known. Several approaches for constrained open-loop MPC based on stochastic models (stochastic MPC) are proposed in [11]-[22]. The approaches [11], [12], [13] are based on linear state space models with stochastic parameters and/or additive noise and they optimize the expected value of the cost function subject to hard input constraints [11] or probabilistic constraints [12], [13]. In [14], [15], [16], [17], stochastic linear MPC approaches incorporating a probabilistic cost and probabilistic constraints are developed. Methods for open-loop stochastic MPC for nonlinear systems have been proposed in [18], [19]. The stochastic MPC approaches [11]-[19] are based on parametric probabilistic models. Alternatively, the stochastic systems can be modeled with non-parametric models, like Gaussian process models. An on-line optimization approach and an approximate explicit approach to open-loop stochastic NMPC based on Gaussian process models have been proposed in [20], [21] and in [22], respectively.

The stochastic MPC methods mentioned above employ an open-loop formulation, which guarantees the robust stability and the robust feasibility of the system, but it may be conservative. This is related to the fact that the control sequence has to ensure constraints fulfillment for all possible uncertainty scenarios without considering the fact that future measurements of the state contain information about past uncertain values. Similar to the closed-loop minmax NMPC approaches [23], [24], [25], the conservativeness of the open-loop stochastic NMPC can be overcome by a closed-loop stochastic NMPC formulation, where the optimization is performed over a sequence of feedback control policies. In [26], [27], methods for closedloop stochastic NMPC based on on-line optimization have been proposed.

Based on the related min-max approximate mp-NLP approach in [28] we consider here the explicit solution of closed-loop (feedback) stochastic NMPC problems for constrained nonlinear systems in the presence of stochastic uncertainty. It is assumed that the discrete probability distribution of the uncertainty is known. The approach constructs a piecewise nonlinear (PWNL) approximation to 
the optimal sequence of feedback control policies for efficient on-line implementation.

\section{Formulation OF THE FEEDBACK StOCHASTIC NMPC PROBLEM AS AN MP-NLP PROBLEM}

Consider the discrete-time nonlinear system:

$$
\begin{aligned}
& x(t+1)=f(x(t), u(t), w(t)) \\
& y(t)=h(x(t), u(t), w(t)),
\end{aligned}
$$

where $x(t) \in \mathfrak{R}^{n}, u(t) \in \mathfrak{R}^{m}, y(t) \in \mathfrak{R}^{s}$ and $w(t) \in \mathfrak{R}^{n}$ are the state, input, output and uncertainty variables, and $t \in \mathbb{Z}_{\geq 0}$ is the discrete time. The input and the output variables are required to fulfill the following constraints:

$$
u_{\text {min }} \leq u \leq u_{\max }, y_{\min } \leq y \leq y_{\max } .
$$

The following assumptions are made:

A1. $f$ and $h$ are $C^{2}$ functions with $f(0,0,0)=0$, $h(0,0,0)=0$.

A2. The uncertainty $w(t)=\left[w_{1}(t), w_{2}(t), \ldots, w_{n}(t)\right]$ includes both internal (state-dependent) and external uncertainty, i.e. it has the form $w_{i}(t)=\lambda_{i} x_{i}(t)+\gamma_{i}(t)$, where $x_{i}(t)$ is the $i$-th element of the state vector $x(t)$. Here $\lambda_{i}$ is a discrete random parameter, associated to the internal (model) uncertainty. It takes values in the set $\Lambda_{i}=\left\{\lambda_{i}^{1}, \lambda_{i}^{2}, \ldots, \lambda_{i}^{n_{\lambda_{i}}}\right\}$ and is characterized with the probability mass function $\varphi_{i}: \Lambda_{i} \rightarrow[0,1]:$

$$
\varphi_{i}\left(\lambda_{i}^{j}\right)=\operatorname{Pr}\left(\lambda_{i}=\lambda_{i}^{j}\right), j=1,2, \ldots, n_{\lambda_{i}} \text { with } \sum_{j=1}^{n_{\lambda_{i}}} \varphi_{i}\left(\lambda_{i}^{j}\right)=1 \text {. }
$$

The external uncertainty $\gamma_{i}(t)$ is stochastic and piecewise constant with infrequent changes in sense that $\gamma_{i}(t)=$ const for periods of time, which are not less than $N_{\gamma}\left(N_{\gamma} \in \mathbb{N}\right.$ is supposed to be sufficiently large). It is assumed that $\gamma_{i}$ takes values in the set $\Gamma_{i}=\left\{\gamma_{i}^{1}, \gamma_{i}^{2}, \ldots, \gamma_{i}^{n_{\gamma_{i}}}\right\}$, which contains the nominal value $\gamma_{i}=0$, and it is characterized with the probability mass function $\psi_{i}: \Gamma_{i} \rightarrow[0,1]$ :

$$
\psi_{i}\left(\gamma_{i}^{j}\right)=\operatorname{Pr}\left(\gamma_{i}=\gamma_{i}^{j}\right), j=1,2, \ldots, n_{\gamma_{i}} \text { with } \sum_{j=1}^{n_{\gamma_{i}}} \psi_{i}\left(\gamma_{i}^{j}\right)=1 \text {. }
$$

The overall vector of uncertain model parameters is denoted $\lambda=\left[\lambda_{1}, \lambda_{2}, \ldots, \lambda_{n}\right] \in \Lambda$ with $\Lambda=\Lambda_{1} \times \Lambda_{2} \times \ldots \times \Lambda_{n}$. Given $\quad \lambda^{j}=\left[\lambda_{1}^{i_{1}}, \lambda_{2}^{i_{2}}, \ldots, \lambda_{n}^{i_{n}}\right], \quad i_{1} \in\left\{1,2, \ldots, n_{\lambda_{1}}\right\}$, $i_{n} \in\left\{1,2, \ldots, n_{\lambda_{n}}\right\}$, the probability mass function $\varphi: \Lambda \rightarrow[0,1]$ is:

$$
\varphi\left(\lambda^{j}\right)=\operatorname{Pr}\left(\lambda=\lambda^{j}\right)=\varphi_{1}\left(\lambda_{1}^{i_{1}}\right) \varphi_{2}\left(\lambda_{2}^{i_{2}}\right) \ldots \varphi_{n}\left(\lambda_{n}^{i_{n}}\right)
$$

with $j=1, \ldots, n_{\lambda}$ and $n_{\lambda}=n_{\lambda_{1}} n_{\lambda_{2}} \ldots n_{\lambda_{n}}$. Similarly, the overall vector of external uncertainty is denoted $\gamma=\left[\gamma_{1}, \gamma_{2}, \ldots, \gamma_{n}\right] \in \Gamma \quad$ with $\Gamma=\Gamma_{1} \times \Gamma_{2} \times \ldots \times \Gamma_{n}$. Given $\gamma^{j}=\left[\gamma_{1}^{i_{1}}, \gamma_{2}^{i_{2}}, \ldots, \gamma_{n}^{i_{n}}\right], i_{1} \in\left\{1,2, \ldots, n_{\gamma_{1}}\right\}, \ldots, i_{n} \in\left\{1,2, \ldots, n_{\gamma_{n}}\right\}$, the probability mass function $\psi: \Gamma \rightarrow[0,1]$ is:

$$
\psi\left(\gamma^{j}\right)=\operatorname{Pr}\left(\gamma=\gamma^{j}\right)=\psi_{1}\left(\gamma_{1}^{i_{1}}\right) \psi_{2}\left(\gamma_{2}^{i_{2}}\right) \ldots \psi_{n}\left(\gamma_{n}^{i_{n}}\right)
$$

with $j=1, \ldots, n_{\gamma}$ and $n_{\gamma}=n_{\gamma_{1}} n_{\gamma_{2}} \ldots n_{\gamma_{n}}$.

Then, the overall uncertainty is $w=\operatorname{diag}(\lambda) x+\gamma$.

A3. $y_{\text {min }}<0<y_{\max }$ and $u_{\text {min }}<0<u_{\text {max }}$.

As in [23], first a $H_{\infty}$ control problem is defined:

Problem P1 ( $H_{\infty}$ control problem):

Design a state-feedback control law:

$$
u=k(x)
$$

guaranteeing that the closed-loop system (1)-(7) with input $w=\operatorname{diag}(\lambda) x+\gamma, \lambda \in \Lambda, \gamma \in \Gamma$, and output $y$ has a finite $l_{2}$ - gain $\leq \alpha$ in a bounded positively invariant set $\Omega$, that is, $\forall x(t) \in \Omega$ :

i. $x(t+i) \in \Omega, \forall i>0$.

ii. $u_{\min } \leq k(x(t+i)) \leq u_{\max }$ and $y_{\min } \leq h(x(t+i), k(x(t+i)), w(t+i)) \leq y_{\max }, \forall i \geq 0$.

iii. There exists a positive definite function $\beta(x(t))$, such that $\forall T \geq 0$ :

$$
\sum_{i=0}^{T}\|y(t+i)\|^{2} \leq \alpha^{2} \sum_{i=0}^{T}\|w(t+i)\|^{2}+\beta(x(t))
$$

for any non-zero $w$.

The following assumption is also made [23]:

A4. There exists an auxiliary control law $u=k_{a}(x)$ that solves the $H_{\infty}$ control problem, with a domain of attraction $\Omega_{a}\left(k_{a}, \alpha, \alpha_{\Delta}\right)$, whose boundary is assumed to be a level curve of a positive function $V_{k_{a}}(x)$ such that:

$$
\begin{aligned}
& V_{k_{a}}\left(f\left(x, k_{a}(x), w\right)\right)-V_{k_{a}}(x)<-\frac{1}{2}\left(\|y\|^{2}-\alpha^{2}\|w\|^{2}\right), \\
& \forall x \in \Omega_{a}\left(k_{a}, \alpha, \alpha_{\Delta}\right), \forall \lambda \in \Lambda, \forall \gamma \in \Gamma
\end{aligned}
$$

and $V_{k_{a}}(0)=0$.

Denote with:

$$
\begin{aligned}
K & =\left\{k_{0}, k_{1}, \ldots, k_{N-1}\right\} \\
& \triangleq\left\{k_{0}\left(x_{t \mid t}\right), k_{1}\left(x_{t+1 \mid t}\right), \ldots, k_{N-1}\left(x_{t+N-1 \mid t}\right)\right\}
\end{aligned}
$$

a vector of feedback control policies. It is supposed that a full measurement $x$ of the state is available at the current time $t$. We consider the following feedback stochastic NMPC problem:

Problem P2 (Constrained feedback stochastic NMPC problem):

Suppose that assumptions A1-A4 hold. For the current $x$, the feedback stochastic NMPC solves the following optimization problem:

$$
V_{E}^{*}(x)=\min _{K} \underset{\lambda \in \Lambda, \gamma \in \Gamma}{E}\{J(K, x, \lambda, \gamma)\}
$$


subject to $x_{t \mid t}=x$ and:

$$
\begin{aligned}
& y_{\min } \leq y_{t+i \mid t} \leq y_{\max }, \forall \lambda \in \Lambda, \forall \gamma \in \Gamma, i=1, \ldots, N \\
& u_{\min } \leq u_{t+i} \leq u_{\max }, i=0,1, \ldots, N-1 \\
& x_{t+N \mid t} \in \Omega_{a}\left(k_{a}, \alpha, \alpha_{\Delta}\right), \forall \lambda \in \Lambda, \forall \gamma \in \Gamma \\
& u_{t+i}=k_{i}\left(x_{t+i \mid t}\right), i=0,1, \ldots, N-1 \\
& x_{t+i+1 \mid t}=f\left(x_{t+i \mid t}, u_{t+i}, w_{t+i}\right), i \geq 0 \\
& y_{t+i \mid t}=h\left(x_{t+i \mid t}, u_{t+i}, w_{t+i}\right), i \geq 0
\end{aligned}
$$

and the cost function given by:

$$
\begin{gathered}
J(K, x, \lambda, \gamma)=\frac{1}{2} \sum_{i=0}^{N-1}\left[\left\|y_{t+i \mid t}\right\|^{2}-\alpha^{2}\left\|w_{t+i}\right\|^{2}\right] \\
+V_{k_{a}}\left(x_{t+N \mid t}\right) .
\end{gathered}
$$

Here, $N$ is a finite horizon, $\alpha$ is the $l_{2}$-gain which is interpreted as the uncertainty attenuation level, and $E\{$. means mathematical expectation. It is supposed that $N \ll N_{\gamma}$, and by Assumption A2 it can be accepted that $\gamma_{t+i}=$ const $, i=0,1, \ldots, N-1$. Then by Assumption A2, the expectation can be expressed:

$$
\underset{\lambda \in \Lambda, \gamma \in \Gamma}{E}\{J(K, x, \lambda, \gamma)\}=\sum_{i=1}^{n_{\lambda}} \sum_{j=1}^{n_{\gamma}} J\left(K, x, \lambda^{i}, \gamma^{j}\right) \varphi\left(\lambda^{i}\right) \psi\left(\gamma^{j}\right)
$$

An auxiliary control law $k_{a}(x)$ is typically obtained by solving the $H_{\infty}$ control problem for the linearized system [23]. Thus, a practical way to compute a nonlinear control $k_{a}(x)$ satisfying assumption A4 for nonlinear input-affine systems is suggested in [23].

An optimal solution to the feedback stochastic NMPC problem (11)-(18) is denoted $K^{*}=\left\{k_{0}^{*}, k_{1}^{*}, \ldots, k_{N-1}^{*}\right\}$ and the control input is chosen according to the receding horizon policy $u\left(x_{t \mid t}\right)=k_{0}^{*}\left(x_{t \mid t}\right)$.

The following assumption is made:

A5. Each feedback control policy $k_{i}\left(x_{t+i \mid t}\right)$, $i=0, \ldots, N-1$ has the form:

$$
k_{i}\left(x_{t+i \mid t}\right)=\eta_{i} k_{a}\left(x_{t+i \mid t}\right)+r_{i}\left(\xi_{i}, x_{t+i \mid t}\right)=g_{i}\left(p_{i}, x_{t+i \mid t}\right),
$$

where $p_{i}=\left[\begin{array}{lll}\eta_{i}^{T} & \xi_{i}^{T}\end{array}\right]^{T} \in \mathfrak{R}^{n_{i}}$ are the parameters that need to be optimized, $k_{a}\left(x_{t+i \mid t}\right)$ is an auxiliary control law that satisfies assumption A4, and $r_{i}\left(\xi_{i}, x_{t+i \mid t}\right)$ is a parameterized continuous function with $r_{i}\left(\xi_{i}, 0\right)=0$.

In general, the parameterization of the form (20) would lead to an approximate solution to the feedback stochastic NMPC problem (11)-(18). Denote with $P$ the whole set of parameters that need to be determined, i.e.:

$$
P=\left[p_{0}^{T} p_{1}^{T} \ldots p_{N-1}^{T}\right]^{T} \in \mathfrak{R}^{n_{p}}, n_{p}=\sum_{i=0}^{N-1} n_{i} .
$$

Then, the expected value (19) of the cost function is:

$$
V_{E}(P, x)=\underset{\lambda \in \Lambda, \gamma \in \Gamma}{E}\{J(P, x, \lambda, \gamma)\} .
$$

It should be noted that the argument $K$ in the cost function (18) is now replaced with the argument $P$.

The optimization problem (11)-(18) can be formulated in a compact form as follows:

\section{Problem P3:}

$$
\begin{aligned}
& V_{E}^{*}(x)=\min _{P} \sum_{i=1}^{n_{\lambda}} \sum_{j=1}^{n_{\gamma}} J\left(P, x, \lambda^{i}, \gamma^{j}\right) \varphi\left(\lambda^{i}\right) \psi\left(\gamma^{j}\right) \\
& \text { subject to: } G(P, x, \lambda, \gamma) \leq 0, \forall \lambda \in \Lambda, \forall \gamma \in \Gamma .
\end{aligned}
$$

Problem P3 defines an mp-NLP, since it is NLP in $P$ parameterized by $x$. We remark that the constraints function $G(P, x, \lambda, \gamma)$ in (24) is implicitly defined by (12)-(17). Also, since $\Lambda$ and $\Gamma$ are finite sets, (24) represents a finite number of constraints. It should be noted that the number of constraints (24) increases rapidly with the increase of the horizon and the sizes $n_{\lambda}$ and $n_{\gamma}$ of the uncertainty sets $\Lambda$ and $\Gamma$. Thus, as the horizon increases from $N_{1}$ to $N_{2}$ and the sizes of the sets $\Lambda$ and $\Gamma$ increase from $n_{\lambda}^{1}$ to $n_{\lambda}^{2}$, and from $n_{\gamma}^{1}$ to $n_{\gamma}^{2}$, respectively, the number of constraints will increase $\left(N_{2} n_{\lambda}^{2} n_{\gamma}^{2}\right) /\left(N_{1} n_{\lambda}^{1} n_{\gamma}^{1}\right)$ times. This leads to a stronger motivation for an explicit approach, where the computational complexity is handled in off-line.

Define the set of $N$-step robustly feasible initial states:

$$
\begin{gathered}
X_{f}=\left\{x \in \mathfrak{R}^{n} \mid G(P, x, \lambda, \gamma) \leq 0, \forall \lambda \in \Lambda, \forall \gamma \in \Gamma\right. \\
\text { for some } \left.P \in \mathfrak{R}^{n_{p}}\right\} .
\end{gathered}
$$

In parametric programming problems one seeks the solution $P^{*}(x)$ as an explicit function of the parameters $x$ in some set $X \subseteq X_{f} \subseteq \mathfrak{R}^{n}[9]$.

\section{APPROXIMATE MP-NLP APPROACH TO EXPLICIT FEEDBACK STOCHASTIC NMPC}

We restrict our attention to a hyper-rectangle $X \subset R^{n}$ where we seek to approximate the optimal sequence of control policies $K^{*}=\left\{k_{0}^{*}, k_{1}^{*}, \ldots, k_{N-1}^{*}\right\}$. The associated optimal control input is $u_{t+i}^{*}=k_{i}^{*}\left(x_{t+i \mid t}\right)=g_{i}\left(p_{i}^{*}, x_{t+i \mid t}\right)$, $i=0,1, \ldots, N-1$, where $\quad P^{*}=\left[\begin{array}{llll}p_{0}^{*} T & p_{1}^{*}{ }^{*} \ldots & p_{N-1}^{*} T\end{array}\right]^{T} \quad$ is determined by solving problem $\mathrm{P} 3$. We require that the state space partition is orthogonal and can be represented as a $k-d$ tree. The main idea of the approximate mp-NLP approach is to construct a piecewise nonlinear (PWNL) approximation $\hat{K}=\left\{\hat{k}_{0}, \hat{k}_{1}, \ldots, \hat{k}_{N-1}\right\} \quad$ to the optimal feedback $K^{*}=\left\{k_{0}^{*}, k_{1}^{*}, \ldots, k_{N-1}^{*}\right\} \quad$ on $X$, where the constituent nonlinear control functions $K_{X_{i}}=\left\{k_{0, X_{i}}, k_{1, X_{i}}, \ldots, k_{N-1, X_{i}}\right\}$ are defined on hyper-rectangles $X_{i}$ covering $X$. Let $K_{X_{0}}=\left\{k_{0, X_{0}}, \ldots, k_{N-1, X_{0}}\right\}$ be an approximation to the optimal solution $K^{*}=\left\{k_{0}^{*}, \ldots, k_{N-1}^{*}\right\}$, valid in the whole 
hyper-rectangle $X_{0}$. Denote with $P_{X_{0}}=\left[\begin{array}{llll}p_{0, X_{0}}^{T} & \ldots & p_{N-1, X_{0}}^{T}\end{array}\right]^{T}$ the parameters of $K_{X_{0}}$. The corresponding approximate value of the control input is $\hat{u}_{t+i}=k_{i, X_{0}}\left(x_{t+i \mid t}\right)=g_{i}\left(p_{i, X_{0}}, x_{t+i \mid t}\right), \quad i=0,1, \ldots, N-1$. Let $\hat{V}_{E}\left(P_{X_{0}}, x\right)$ be the cost function value due to initial state $x=x_{t \mid t} \quad$ and control function $\quad K_{X_{0}}$, i.e. $\hat{V}_{E}\left(P_{X_{0}}, x\right)=\underset{\lambda \in \Lambda, \gamma \in \Gamma}{E}\left\{J\left(P_{X_{0}}, x, \lambda, \gamma\right)\right\} . \quad$ Then, the approximate control function $K_{X_{0}}$ is determined by applying the following procedure:

\section{Procedure 1:}

Suppose A1-A5 hold. Consider any hyper-rectangle $X_{0} \subseteq X_{f}$ with a set of points $V_{0}=\left\{v_{0}, v_{1}, v_{2}, \ldots, v_{N_{1}}\right\}$. Compute the parameters $P_{X_{0}}=\left[\begin{array}{llll}p_{0, X_{0}}^{T} & \ldots & p_{N-1, X_{0}}^{T}\end{array}\right]^{T}$ of the control function $K_{X_{0}}=\left\{k_{0, X_{0}}, \ldots, k_{N-1, X_{0}}\right\}$ by solving the following NLP:

$$
\begin{aligned}
\min _{P_{X_{0}}} \sum_{i=0}^{N_{1}}\left(\hat{V}_{E}\left(P_{X_{0}}, v_{i}\right)-V_{E}^{*}\left(v_{i}\right)\right. & \\
& \left.+\mu\left\|g_{0}\left(p_{0, X_{0}}, v_{i}\right)-g_{0}\left(p_{0}^{*_{i}}, v_{i}\right)\right\|^{2}\right)
\end{aligned}
$$

subject to:

$$
G\left(P_{X_{0}}, v_{i}, \lambda, \gamma\right) \leq 0, \forall v_{i} \in V_{0}, \forall \lambda \in \Lambda, \forall \gamma \in \Gamma .
$$

We remark that the optimal parameters $p_{0}^{*_{i}}$ in the feedback function $k_{0}^{*}\left(v_{i}\right)=g_{0}\left(p_{0}^{*_{i}}, v_{i}\right)$ in (26) are determined by solving problem P3 for $x=v_{i}$ and the parameter $\mu>0$ is a weighting coefficient.

Note that the control function $K_{X_{0}}=\left\{k_{0, X_{0}}, \ldots, k_{N-1, X_{0}}\right\}$, computed with Procedure 1, satisfies the constraints in problem P3 only for the discrete set of points $V_{0}$ in the hyper-rectangle $X_{0}$ and for the discrete sets of values $\Lambda$ and $\Gamma$ of the internal and external uncertainties.

Suppose that the parameter vector $P_{X_{0}}$ of the control function $K_{X_{0}}$, valid in $X_{0}$, has been determined by applying Procedure 1. Then, for the cost function approximation error in $X_{0}$ we have:

$$
\varepsilon(x)=\hat{V}_{E}\left(P_{X_{0}}, x\right)-V_{E}^{*}(x) \leq \varepsilon_{0}, x \in X_{0} .
$$

An estimate $\hat{\varepsilon}_{0}$ of the error bound $\varepsilon_{0}$ is computed as:

$$
\hat{\varepsilon}_{0}=\max _{i \in\left\{0,1,2, \ldots, N_{1}\right\}}\left(\hat{V}_{E}\left(P_{X_{0}}, v_{i}\right)-V_{E}^{*}\left(v_{i}\right)\right) .
$$

If $\hat{\varepsilon}_{0}>\bar{\varepsilon}$, where $\bar{\varepsilon}>0$ is the specified tolerance of the cost function approximation error, the region $X_{0}$ is divided and the procedure is repeated for the new regions. The detailed approximate mp-NLP algorithm is given in [28].

It should be noted that in case of non-convexity of problem P3, it can not be guaranteed that the approximation error $\varepsilon(x)$ associated to the explicit feedback stochastic NMPC will satisfy the requirement $\varepsilon(x) \leq \bar{\varepsilon}$ for all $x \in X$. The non-convexity may also imply that the constraints (24) are violated at some points of the state space. In this respect, the described computational method does not necessarily lead to guaranteed properties, but when combined with verification and analysis methods gives a practical tool for development and implementation of explicit feedback stochastic NMPC. The possibility for implementation verification is a significant advantage of the explicit NMPC in comparison to NMPC based on real-time optimization.

\section{Simulation EXAMPLE}

Consider a cart with a mass $M$ moving on a plane [23]. The carriage is attached to the wall via a spring with elasticity $\rho=\rho_{0} e^{-x_{1}}$, where $x_{1}$ is the displacement of the carriage from the equilibrium position associated with the external force $u=0$ [23]. A damper with damping factor $h_{d}$ affects the system in a resistive way. The damping factor $h_{d}$ is uncertain, but it is known that $h_{d}=\bar{h}_{d}+\lambda$. Here, $\bar{h}_{d}=1.1$ and $\lambda$ is a stochastic parameter. It is supposed that $-0.5 \leq \lambda \leq 0.5$ and the following discrete set of values is considered $\lambda \in \Lambda=\{-0.5,0,0.5\}$ with the corresponding values of the probability mass function $\varphi(-0.5)=0.2$, $\varphi(0)=0.6, \varphi(0.5)=0.2$. The external uncertainty for this system is $\gamma=0$. The system is described by the following nonlinear discrete-time model [23]:

$$
\begin{aligned}
x_{1}(t+1)=x_{1}(t) & +T_{s} x_{2}(t) \\
x_{2}(t+1)=x_{2}(t) & -T_{s} \frac{\rho_{0}}{M} e^{-x_{1}(t)} x_{1}(t)-T_{s} \frac{\bar{h}_{d}}{M} x_{2}(t) \\
& +T_{s} \frac{u(t)}{M}+T_{s} w(t),
\end{aligned}
$$

where $x_{2}$ is the carriage velocity, $w(t)=-\frac{\lambda}{M} x_{2}(t)$ is a state dependent (internal) uncertainty, $T_{s}=0.4$ is the sampling time, $M=1$ and $\rho_{0}=0.33$. The following input and state constraints are imposed on the system:

$$
-4 \leq u \leq 4,-1.3 \leq x_{2} \leq 1.3 \text {. }
$$

The horizon is $N=15$ and the terminal constraint is:

$$
x_{t+N \mid t} \in \Omega_{a}, \Omega_{a}=\left\{x \in \mathfrak{R}^{n} \mid x^{T} \Sigma x \leq \delta\right\},
$$

where $\delta=0.001$ [23] and $\Sigma=\left[\begin{array}{ll}1.3 & 1.9 \\ 1.9 & 3.0\end{array}\right]$.

The mp-NLP approach described in section III is applied to design an explicit feedback stochastic NMPC controller for the cart. The NMPC minimizes the mathematical expectation (19) of the cost function (18) subject to the system equations (30), (31) and the constraints (32)-(33). In (18), it is chosen $\alpha=1$ and the terminal penalty is given by 
$V_{k_{a}}=x^{T} \Sigma x$ [23]. Like in [23], the feedback functions $k_{i}\left(x_{t+i \mid t}\right), i=0, \ldots, N-1$ have the form:

$$
k_{i}\left(p_{i}, x_{t+i \mid t}\right)=\eta_{i} k_{a}\left(x_{t+i \mid t}\right)+\xi_{i, 1} x_{1, t+i \mid t}^{2}+\xi_{i, 2} x_{2, t+i \mid t}^{2},
$$

where $p_{i}=\left[\eta_{i} \xi_{i, 1} \xi_{i, 2}\right]^{T}$ are the parameters that need to be optimized and $k_{a}\left(x_{t+i \mid t}\right)$ is the auxiliary control law. The expression (34) implies that for relatively small absolute deviations from the equilibrium (small $x_{1, t+i \mid t}^{2}$ and $x_{2, t+i \mid t}^{2}$ ) the control input value will be generated mainly by the auxiliary control law $k_{a}\left(x_{t+i \mid t}\right)$. The control law $k_{a}\left(x_{t+i \mid t}\right)$ is determined by applying the method in [23]:

$$
k_{a}\left(x_{t+i \mid t}\right)=-\left[\begin{array}{ll}
1 & 0
\end{array}\right] R^{-1}\left[\begin{array}{l}
F_{2}^{T} \\
F_{3}^{T}
\end{array}\right] \Sigma f_{1}\left(x_{t+i \mid t}\right),
$$

where:

$$
\begin{gathered}
f_{1}\left(x_{t+i \mid t}\right)=\left[\begin{array}{c}
x_{1, t+i \mid t}+T_{s} x_{2, t+i \mid t} \\
x_{2, t+i \mid t}-T_{s} \frac{\rho_{0}}{M} e^{-x_{1, t+i \mid t}} x_{1, t+i \mid t}-T_{s} \frac{\bar{h}_{d}}{M} x_{2, t+i \mid t}
\end{array}\right] \\
F_{2}=\left[\begin{array}{c}
0 \\
\frac{T_{s}}{M}
\end{array}\right], F_{3}=\left[\begin{array}{c}
0 \\
T_{s}
\end{array}\right], R=\left[\begin{array}{cc}
F_{2}^{T} \Sigma F_{2}+I & F_{2}^{T} \Sigma F_{3} \\
\left(F_{2}^{T} \Sigma F_{3}\right)^{T} & F_{3}^{T} \Sigma F_{3}-\alpha^{2} I
\end{array}\right]
\end{gathered}
$$

In [7], a condition on the approximation tolerance has been derived such that the asymptotic stability of the nonlinear system in closed-loop with the approximate explicit NMPC is guaranteed. According to this condition, the tolerance is chosen to be dependent on the state, which would lead to a state space partition with less complexity in comparison to that corresponding to an uniform tolerance. Here, a similar approach is applied and the approximation tolerance is chosen to be dependent on the state as $\bar{\varepsilon}\left(X_{0}\right)=\max \left(\bar{\varepsilon}_{a}, \bar{\varepsilon}_{r} \min _{x \in X_{0}} V_{E}^{*}(x)\right)$, where $\bar{\varepsilon}_{a}=0.005$ and $\bar{\varepsilon}_{r}=0.03$ are the absolute and the relative tolerances.

The state space partition of the explicit feedback stochastic NMPC controller is shown in Fig. 1.

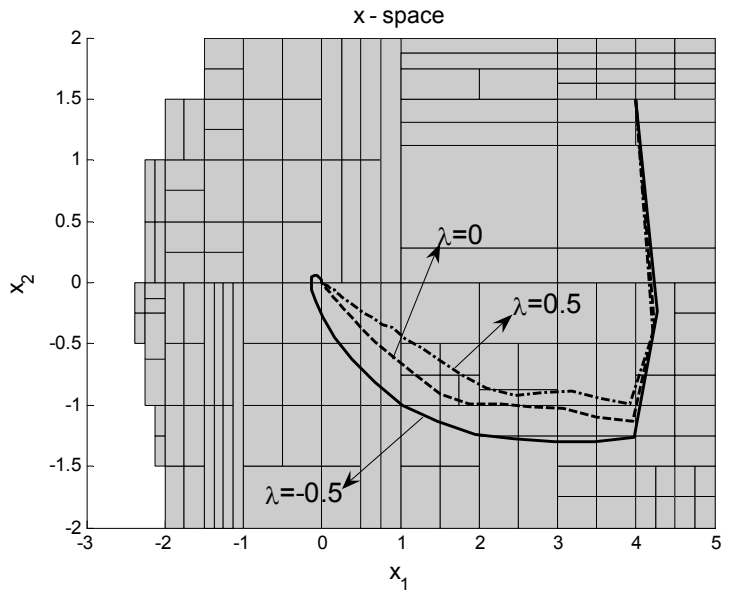

Fig. 1. State space partition of the explicit feedback stochastic NMPC and the state trajectories corresponding to $\lambda=-0.5, \lambda=0, \lambda=0.5$.
The partition has 150 regions and 11 levels of search. Totally, 27 arithmetic operations are needed in real-time to compute the control input (11 comparisons, 10 multiplications, 5 additions and 1 exponential).

In Fig. 2, the suboptimal feedback function $\hat{u}\left(x_{1}, x_{2}\right)=\hat{k}_{0}\left(x_{1}, x_{2}\right)$ is shown.

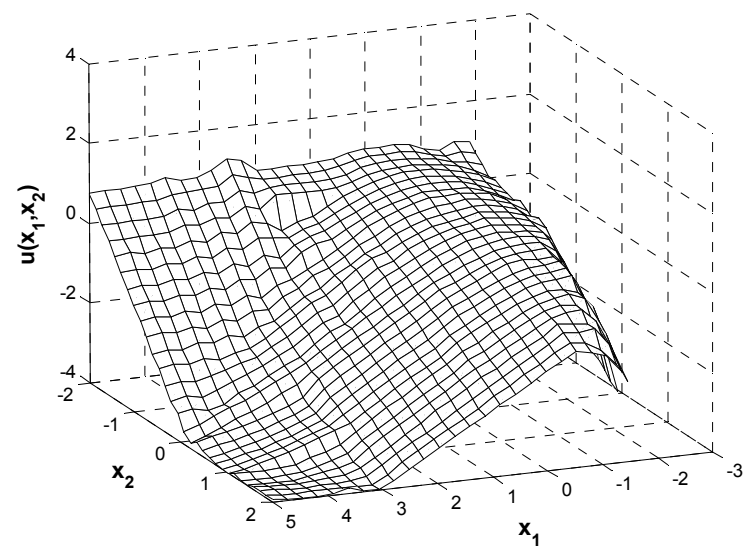

Fig. 2. The suboptimal feedback function $\hat{u}\left(x_{1}, x_{2}\right)=\hat{k}_{0}\left(x_{1}, x_{2}\right)$ (view rotated on $140^{\circ}$ ).

The performance of the closed-loop system was simulated for initial state $x(0)=\left[\begin{array}{ll}4 & 1.5\end{array}\right]^{T}$ and for the three values of the stochastic parameter $\lambda$. The response is depicted in the state space (Fig. 1), as well as trajectories in time (Fig. 3 and Fig. 4). In Fig. 3 and Fig. 4, the control and state trajectories obtained with the explicit min-max NMPC controller [28] are given for comparison. The cost function values corresponding to the closed-loop trajectories associated to the explicit stochastic NMPC and to the explicit min-max $\mathrm{NMPC}$ are $\hat{V}_{E}=121.64$ and $\hat{V}_{\min -\max }=122.57$ (for $h_{d}=0.6$ ), and $\hat{V}_{E}=141.59$ and $\hat{V}_{\min -\max }=141.70$ (for $\left.h_{d}=1.6\right)$. Therefore, the explicit min-max NMPC is slightly more conservative, since it is characterized with larger values of the cost function in comparison to the explicit stochastic NMPC.
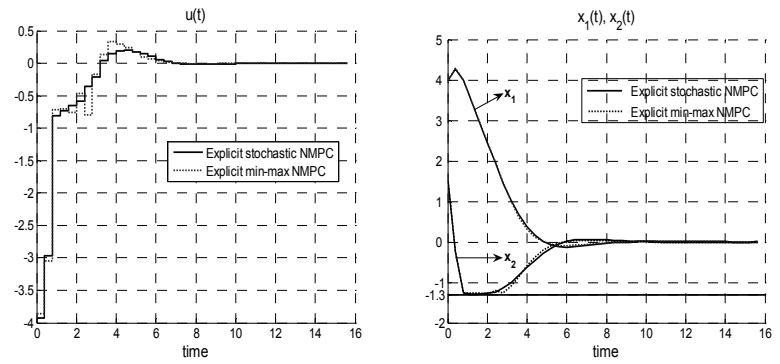

Fig. 3. Control input (left) and state trajectory (right) for $h_{d}=0.6$. 

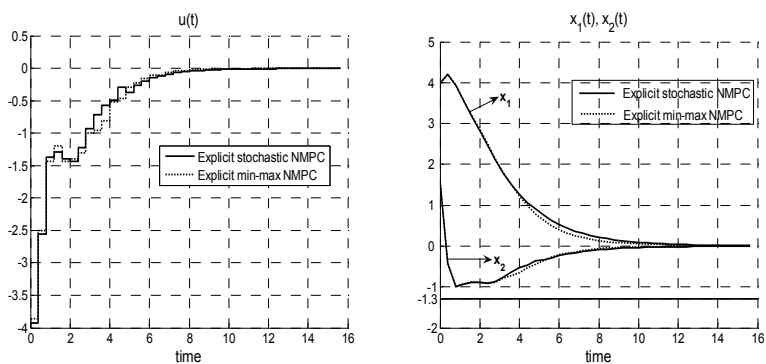

Fig. 4. Control input (left) and state trajectory (right) for $h_{d}=1.6$.

It can be seen that the explicit feedback stochastic NMPC controller brings the cart to the equilibrium despite of the presence of stochastic uncertainty, and the constraints imposed on the system are satisfied.

\section{CONCLUSION}

This paper presents an approximate mp-NLP approach to explicit solution of feedback stochastic MPC problems for constrained nonlinear systems in the presence of stochastic uncertainty. It is applied to design an explicit feedback stochastic NMPC for a cart moving on a plane and attached to the wall via a spring. Results show that the explicit stochastic NMPC controller brings the cart to the equilibrium despite of the presence of stochastic uncertainty, and the constraints imposed on the system are satisfied.

\section{REFERENCES}

[1] D. Q. Mayne, J. B. Rawlings, C. V. Rao, and P. O. M. Scokaert, "Constrained model predictive control: Stability and optimality", Automatica, vol. 36, pp. 789-814, 2000.

[2] F. Allgöwer and A. Zheng (eds.), Nonlinear Model Predictive Control, Progress in system and control theory, Vol. 26, Basel: Birkhäuser Verlag, 2000.

[3] B. Kouvaritakis and M. Cannon (eds.), Nonlinear predictive control, Theory and practice, IEE Control Engineering Series 61, IEE, 2001.

[4] M. Diehl, H. J. Ferreau, and N. Haverbeke, "Efficient numerical methods for nonlinear MPC and moving horizon estimation", in: L. Magni, D. M. Raimondo, and F. Allgöwer (Eds.), Nonlinear Model Predictive Control: Towards New Challenging Applications, LNCIS, vol. 384, Berlin/Heidelberg: Springer-Verlag, 2009, pp.391-418.

[5] V. M. Zavala and L. T. Biegler, "The advanced-step NMPC controller: Optimality, stability and robustness", Automatica, vol. 45 pp. 86-93, 2009.

[6] T. A. Johansen, "On multi-parametric nonlinear programming and explicit nonlinear model predictive control", Proceedings of IEEE Conference on Decision and Control, Las Vegas, NV, 2002, vol. 3, pp. 2768-2773.

[7] T. A. Johansen, "Approximate explicit receding horizon control of constrained nonlinear systems", Automatica, vol. 40, pp. 293-300, 2004.

[8] A. Grancharova, T. A. Johansen, and P. Tøndel, "Computational aspects of approximate explicit nonlinear model predictive control", in R. Findeisen, F. Allgöwer and L. Biegler (Eds.), Assessment and Future Directions of Nonlinear Model Predictive Control, LNCIS, vol. 358, Springer-Verlag, Germany, pp. 181-192, 2007.

[9] A. V. Fiacco, Introduction to sensitivity and stability analysis in nonlinear programming. Orlando, Fl: Academic Press, 1983.

[10] E. N. Pistikopoulos, M. C. Georgiadis, and V. Dua, Multi-parametric Programming: theory, algorithms, and applications, Wiley- $\mathrm{VCH}$, 2007.
[11] J. H. Lee and B. L. Cooley, "Optimal feedback control strategies for state-space systems with stochastic parameters", IEEE Transactions on Automatic Control, vol. 43, No. 10, pp. 1469-1475, 1998.

[12] D. van Hessem, C. W. Scherer, and O. H. Bosgra, "LMI-based closed-loop economic optimization of stochastic process operation under state and input constraints", in Proc. of the 40-th IEEE Conference on Decision and Control, 2001, pp. 4228-4233.

[13] J. Yan and R. R. Bitmead, "Incorporating state estimation into model predictive control and its application to network traffic control", Automatica, vol. 41, pp. 595-604, 2005.

[14] B. Kouvaritakis, M. Cannon, and P. Couchman, "MPC as a tool for sustainable development integrated policy assessment", IEEE Transactions on Automatic Control, vol. 51, pp. 145-149, 2006.

[15] P. Couchman, B. Kouvaritakis, and M. Cannon, "LTV models in MPC for sustainable development", International Journal of Control, vol. 79, pp. 63-73, 2006.

[16] P. Couchman, M. Cannon, and B. Kouvaritakis, "Stochastic MPC with inequality stability constraints", Automatica, vol. 42, pp. 2169-2174, 2006.

[17] M. Cannon, P. Couchman, and B. Kouvaritakis, "MPC for stochastic systems", in R. Findeisen, F. Allgöwer and L. Biegler (Eds.), Assessment and Future Directions of Nonlinear Model Predictive Control, LNCIS, vol. 358, Berlin/Heidelberg: Springer-Verlag, pp. 255-268, 2007.

[18] M. Cannon, D. Ng and B. Kouvaritakis, "Successive linearization NMPC for a class of stochastic nonlinear systems", in L. Magni, D. M. Raimondo and F. Allgöwer (Eds.), Nonlinear Model Predictive Control: Towards New Challenging Applications, LNCIS, vol. 384, pp. 249-262, Berlin/Heidelberg: Springer-Verlag, 2009.

[19] N. Kantas, J. M. Maciejowski, and A. Lecchini-Visintini, "Sequential Monte Carlo for model predictive control", in L. Magni, D. M. Raimondo and F. Allgöwer (Eds.), Nonlinear Model Predictive Control: Towards New Challenging Applications, LNCIS, vol. 384 pp. 263-274, Berlin/Heidelberg: Springer-Verlag, 2009.

[20] R. Murray-Smith, D. Sbarbaro, C. E. Rasmussen, and A. Girard, "Adaptive, cautious, predictive control with Gaussian process priors", in Proc. of 13-th IFAC Symposium on System Identification, Rotterdam, 2003.

[21] B. Likar and J. Kocijan, "Predictive control of a gas-liquid separation plant based on a Gaussian process model", Computers \& Chemical Engineering, vol. 31, pp. 142-152, 2007.

[22] A. Grancharova, J. Kocijan, and T. A. Johansen, "Explicit stochastic nonlinear predictive control based on Gaussian process models", in Proc. of the European Control Conference, Kos, Greece, 2007, pp. 2340-2347.

[23] L. Magni, G. De Nicolao, R. Scattolini, and F. Allgöwer, "Robust model predictive control for nonlinear discrete-time systems", International Journal of Robust and Nonlinear Control, vol. 13, pp. 229-246, 2003.

[24] D. Limon, T. Alamo, F., Salas, and E. F. Camacho, "Input-to-state stability of min-max MPC controllers for nonlinear systems with bounded uncertainties", Automatica, vol. 42, pp. 797-803, 2006.

[25] L. Magni and R. Scattolini, "Robustness and robust design of MPC for nonlinear discrete-time systems", in R. Findeisen, F. Allgöwer, and L. Biegler (Eds.), Assessment and future directions of nonlinear model predictive control, LNCIS, vol. 358, pp. 239-254, Springer-Verlag, 2007.

[26] G. C. Goodwin, J. Østergaard, D. E. Quevedo, and A. Feuer, "A vector quantization approach to scenario generation for stochastic NMPC", in L. Magni, D. M. Raimondo and F. Allgöwer (Eds.), Nonlinear Model Predictive Control: Towards New Challenging Applications, LNCIS, vol. 384, pp. 235-248, Berlin/Heidelberg: Springer-Verlag, 2009.

[27] H. Arellano-Garcia, M. Wendt, T. Barz, and G. Wozny, "Close-loop stochastic dynamic optimization under probabilistic outputconstraints", in R. Findeisen, F. Allgöwer, and L. Biegler (Eds.), Assessment and future directions of nonlinear model predictive control, LNCIS, vol. 358, pp. 305-315, Springer-Verlag, 2007.

[28] A. Grancharova and T. A. Johansen, "Computation, approximation and stability of explicit feedback min-max nonlinear model predictive control", Automatica, vol. 45, pp. 1134-1143, 2009. 